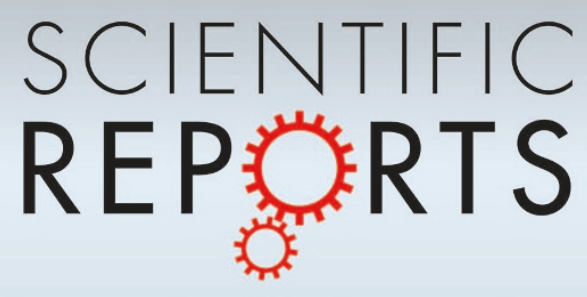

OPEN

SUBJECT AREAS:

SURFACES, INTERFACES

AND THIN FILMS

TWO-DIMENSIONAL MATERIALS

SEMICONDUCTORS

Received

28 July 2014

Accepted

24 February 2015

Published

18 March 2015

Correspondence and requests for materials should be addressed to

X.C. (xdcui@hku.hk)

* These authors contributed equally to this work.

\section{Exciton Binding Energy of Monolayer} $\mathrm{WS}_{2}$

\author{
Bairen Zhu*, Xi Chen* \& Xiaodong Cui
}

Physics Department, University of Hong Kong, Hong Kong, China.

The optical properties of monolayer transition metal dichalcogenides (TMDC) feature prominent excitonic natures. Here we report an experimental approach to measuring the exciton binding energy of monolayer $\mathrm{WS}_{2}$ with linear differential transmission spectroscopy and two-photon photoluminescence excitation spectroscopy (TP-PLE). TP-PLE measurements show the exciton binding energy of $0.71 \pm 0.01 \mathrm{eV}$ around $\mathrm{K}$ valley in the Brillouin zone.

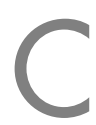

oulomb interactions are significantly enhanced in low dimensional systems as a result of spatial confinement and reduced Coulomb screening. Consequently, excitons, quasiparticles of electron-hole pairs bounded by Coulomb force, play a pronounced role in their optical aspects. A few paradigms of the pronounced excitonic effects have been demonstrated in quantum dots and carbon nanotubes, where the exciton binding energies are found to be a fraction of their band gaps in these quasi-zero dimensional (0D) and one dimensional (1D) systems ${ }^{1-3}$. Prominent exciton effects are also widely expected in intrinsic 2D systems, for instance, monolayer crystals of transition metal dichalcogenides (TMDCs) owing to the reduced dielectric screening and spatial confinements ${ }^{4-8}$. Monolayer TMDC is an intrinsic $2 \mathrm{D}$ crystal consisting of two hexagonal planes of chalcogen atoms and an intermediate hexagonal plane of metal atoms in a prismatic unit cell. Particularly $\mathrm{MX}_{2}\left(\mathrm{MoS}_{2}, \mathrm{MoSe}_{2}, \mathrm{WS}_{2}\right.$ and $\left.\mathrm{WSe}_{2}\right)$ experiences a transition from indirect gap in bulk form to direct gap of visible frequency in monolayer, where the band gap is located at $\mathrm{K}\left(\mathrm{K}^{\prime}\right)$ valley of the Brillouin zone $^{9-12}$. Ab initio calculations show the direct-gap exciton binding energy in the range of $0.5-1 \mathrm{eV}$, which is around $1 / 3-1 / 2$ of the corresponding optical direct gap ${ }^{4-8}$. The modulated absorption/reflection spectroscopy shows the binding energy of direct gap exciton is around $55 \mathrm{meV}$ in bulk crystals ${ }^{13}$. Such a big exciton binding energy in bulk form guarantees the robust excitonic nature of optical properties in ultrathin counterparts. Furthermore, photoluminescence (PL) experiments identify electron (hole)-bounded excitons, so called trions, with a charging energy $E_{b X-}$ of $18 \mathrm{meV}, 30 \mathrm{meV}$ and $20-40 \mathrm{meV}$ in monolayer $\mathrm{MoS}_{2}, \mathrm{MoSe}_{2}$ and $\mathrm{WS}_{2}$, respectively ${ }^{14-16}$. With a simple 2D exciton model, one could estimate the exciton binding energy around 10 times that of the trion, if equal effective electron's and hole's mass are assumed ${ }^{17}$. However, the direct measurement of the exciton binding energy in monolayer TMDC is lacking (After posting of this work, the experimental studies ${ }^{18-24}$ on exciton binding energy and exciton excited states in monolayer TMDCs have been reported).

Here we report experimental approaches to measuring the exciton binding energy of monolayer $\mathrm{WS}_{2}$ with linear differential transmission spectroscopy and two-photon photoluminescence excitation spectroscopy (TPPLE). The TP-PLE resolves the excited states of excitons and the interband transition continuum. The exciton binding energy of $0.71 \pm 0.01 \mathrm{eV}$ of the band-edge excitons around $\mathrm{K}$ valley in the Brillouin zone is extracted by the energy difference between the ground state exciton and the onset of the interband continuum.

Monolayer $\mathrm{WS}_{2}$ was mechanically exfoliated from single crystal $\mathrm{WS}_{2}$ and identified with optical microscopes and photoluminescence spectroscopy (supplementary information). The samples in differential transmission measurements were made by transferring from silicon substrates to freshly cleaved mica substrates as described in Ref. 25. All other measurements were performed on $\mathrm{WS}_{2}$ monolayers on silicon substrates with a $300 \mathrm{~nm}$ oxide cap layer. The electric-gate dependent PL measurements were conducted with a back-gated field effect transistor structure with Au electrodes. The TP-PLE spectroscopy was undertaken at ambient conditions by deploying a confocal setup with a $20 \times$ achromatic objective and a Ti:sapphire oscillator ( $80 \mathrm{MHz}, 100 \mathrm{fs})$.

Figure 1(a) summarizes linear optical measurements of monolayer and multilayer $\mathrm{WS}_{2}$. There are distinct peaks in the differential transmission spectra, labeled as " $A$ ", " $B$ " and " $C$ ", respectively" 12,13 . Peaks " $A$ " around $2.02 \mathrm{eV}$ and " $\mathrm{B}$ " around $2.4 \mathrm{eV}$ at room temperature present the excitonic absorptions of the direct gap located at $\mathrm{K}$ valley of the Brillouin zone. The separation between " $\mathrm{A}$ " and " $\mathrm{B}$ " of $0.38 \mathrm{eV}$ rising from the splitting of the valence band minimum (VBM) due to the spin-orbit coupling (SOC) at $\mathrm{K}\left(\mathrm{K}^{\prime}\right)$ valley is almost constant in all the 


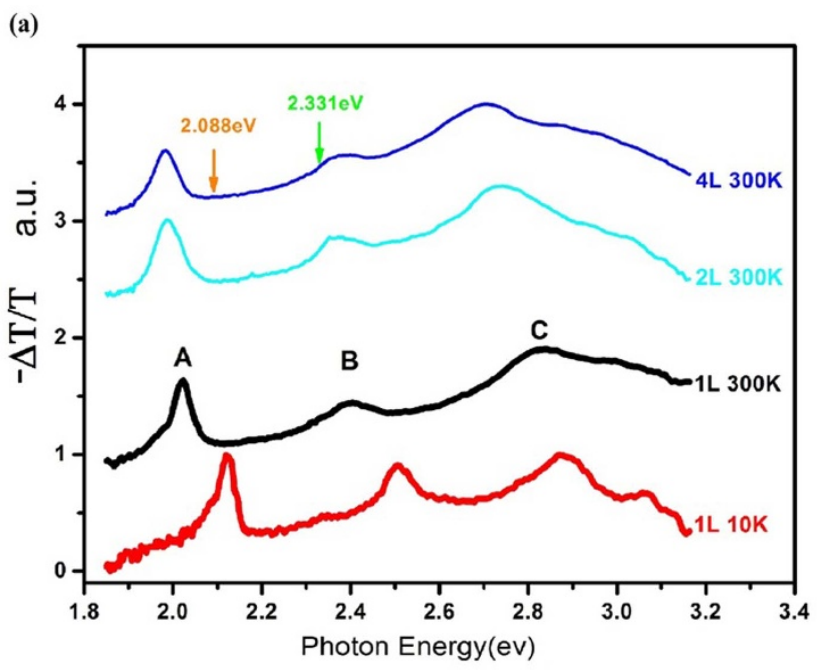

(b)

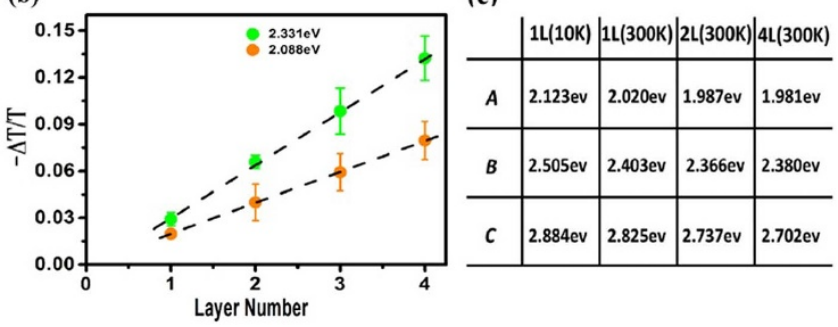

Figure 1 The linear absorption spectra of $\mathrm{WS}_{2}$ atomically thin films. (a) Normalized differential transmission spectra of multi- and monolayer $\mathrm{WS}_{2}$ at room temperature and $10 \mathrm{~K}$. (b) Absorbance of atomically thin layer at photon energy of $2.088 \mathrm{eV}$ (orange) and $2.331 \mathrm{eV}$ (green) as indicated by arrows in figure 1 (a). The absorbance shows a linearly dependence on layer number, each unit layer with constants of $2.0 \%$ and $3.4 \%$ respectively. (c) Absorption peak energy values of exciton " $A$ ", " $B$ " and "C".

layers with various thickness, which is consistent with the PL spec$\operatorname{tra}^{11,12}$. It is the direct result of the suppression of interlayer coupling at $\mathrm{K}\left(\mathrm{K}^{\prime}\right)$ valley owing to the giant SOC and spin-valley coupling in tungsten TMDC with $2 \mathrm{H}$ stacking order, in which each unit layer is a $\pi$ rotation of its adjacent layers ${ }^{11}$. The peak " $\mathrm{C}$ " around $2.8 \mathrm{eV}$ was recognized as the excitonic transitions from multiple points near $\Gamma$ point of the Brillouin zone ${ }^{5,12}$. Unlike in many semiconductors, the linear absorption spectra of $\mathrm{WS}_{2}$ display no gap between distinct excitons and the continuum of the interband transitions. In addition to the overlap with the higher-energy states (exciton C), the continuous absorption may also originate from the strong electron (hole)phonon coupling and the superposition of high-energy excited states of excitons (exciton A and exciton $\mathrm{B}$ ) that fill the gap between the ground state excitons and the interband continuum in the linear absorption spectra ${ }^{5,19}$. As the temperature drops to $10 \mathrm{~K}$, peak "A" and " $\mathrm{B}$ " are both blue-shifted by around $0.1 \mathrm{eV}$ and peak " $\mathrm{C}$ " is shifted by $0.06 \mathrm{eV}$ as shown in Figure 1(c). The difference of the blue-shift is the direct consequence of the diverse locations of the excitons in the Brillouin zone: exciton " $\mathrm{A}$ " and "B" are formed at $\mathrm{K}$ valley while " $C$ " is around $\Gamma$ point. Nevertheless, the continuous absorption still survives and no distinct single-particle band edge emerges at cryogenic temperature $(10 \mathrm{~K})$. It is difficult to resolve the exciton binding energy by the linear absorption spectra owing to the overlap of high-energy excitons (exciton $\mathrm{C}$ ), the strong electron-phonon coupling and the transfer of the oscillator strength from interband continuum to exciton states. The strong electronphonon coupling and the transfer of the oscillator strength are the direct consequences of enhanced coulomb interactions in low dimensional materials as reflected in carbon nanotubes ${ }^{2,26}$.
Figure 1(b) shows the absorbance of atomically thin $\mathrm{WS}_{2}$ films as a function of the thickness above their ground exciton energy, which is approximated with the differential transmission. The absorbance of monolayers and multilayers is linearly proportional to their thickness, each layer absorbing around $2.0 \%$ and $3.4 \%$ at excitations of $2.088 \mathrm{eV}$ and $2.331 \mathrm{eV}$, respectively. The linear layer dependence of the absorption gives an experimental evidence of the suppression of interlayer hopping in $2 \mathrm{H}$ stacked $\mathrm{WS}_{2}$ as a result of spin-valley coupling ${ }^{11,27}$. The thickness dependence could also be used as a thickness monitor for multilayer characterization. There is a side band at the red side of exciton A, which modifies the lineshape away from the symmetric Lorentzian or Gaussian shape. We tentatively attribute the side band to the contribution of electron/hole-bound excitons or trions ${ }^{14-16}$. Although the monolayer $\mathrm{WS}_{2}$ is not intentionally doped, the structural defects and substrate effects such as charge transfer and defects modulate the carrier density away from its insulating state. To confirm the origin of the side band around exciton A, we record the PL spectra of monolayer $\mathrm{WS}_{2}$ at various electric gating (from $70 \mathrm{~V}$ to $-70 \mathrm{~V}$ ) at room temperature in vacuum, which continuously tunes the Fermi level of monolayer $\mathrm{WS}_{2}$ as illustrated in Figure 2(a).

There is a prominent peak $\mathrm{X}^{-}$at the red side of the free exciton $\mathrm{X}^{0}$ at $\mathrm{V}_{\mathrm{g}} \approx-20 \mathrm{~V}$ and the PL spectrum could be described by a superposition of two Lorentzian fittings which center at peak $\mathrm{X}^{0}$ and $\mathrm{X}^{-}$ respectively as illustrated in Figure 2(b). As the gate voltage goes towards positive values $\left(\mathrm{V}_{\mathrm{g}}>0\right)$, the free exciton $\mathrm{X}^{0}$ gradually diminishes and disappears at $\mathrm{Vg}>40$. Meanwhile, the red-side $\mathrm{X}^{-}$ rises to take over the overwhelming weight of the whole PL until it starts to decrease at $\mathrm{V}_{\mathrm{g}}>20$, probably due to the electrostatic screening effect ${ }^{28}$, and the peak $\mathrm{X}^{-}$is further red-shifted. The electric gating dependence attributes $\mathrm{X}^{-}$to $\mathrm{n}$-type trion (electron-bounded exciton) states. As $\mathrm{V}_{\mathrm{g}}$ goes to negative bias, the free exciton state $\mathrm{X}^{0}$ takes over the weight of the PL and tends to saturate around $\mathrm{V}_{\mathrm{g}}=-70 \mathrm{~V}$. While the trion state $\mathrm{X}^{-}$monotonically diminishes, the redshift also shows a sign of saturation of $-34 \mathrm{meV}$ at around $\mathrm{V}_{\mathrm{g}}=-70 \mathrm{~V}$. This confirms the trion (electron-bound exciton) origin of the side band around exciton $\mathrm{A}$ in the monolayer transmission spectrum and the trion binding energy of $34 \mathrm{meV}$ in monolayer. If we follow the simplified trion model in conventional quantum wells ${ }^{17}$ and take the effective mass of either $m_{e}=0.37$ and $m_{h}=-0.48^{29}$ or $m_{e}=0.27$, $m_{h}=-0.32^{7}$, the binding energy of free exciton is estimated at $E_{b} \approx$ $0.34 \mathrm{eV}$. We will show this estimation is oversimplified in the following part.

Two-photon excitation is a third order optical process involving simultaneous absorption of the two photons, which follows selection rules different from those in one-photon (linear) process. As a photon has an odd intrinsic parity, one- and two-photon transitions are mutually exclusive in systems with inversion symmetry: onephoton transitions are allowed between the states with different parities, while two-photon transitions between the states with the same parity. In systems without inversion symmetry like monolayer TMDCs described by a point group of $D_{3 h}$ symmetry, parity is not a good quantum number and there exist transitions which are both one- and two-photon allowed. Nevertheless, the oscillator strengths of exciton states are generally different between one- and two-photon processes. A simplified exciton model could be described as $U_{n}^{l}\left(\rho=r_{e}-r_{h}\right) \varphi_{c}\left(r_{e}\right) \varphi_{v}\left(r_{h}\right)$, where $\varphi_{c}\left(r_{e}\right)\left(\varphi_{v}\left(r_{h}\right)\right)$ presents the electron (hole) wave function, and $U_{n}^{l}$ is the function of the relative motion of electron-hole. The optical transition rates for one- and two-photon processes ${ }^{30}$

$$
\begin{aligned}
& W_{O P} \sim|A|^{2} \sum_{c, v}|\langle c|\varepsilon \cdot p| v\rangle|^{2} \sum_{c v}\left|\left\langle\varphi_{c} \mid \varphi_{h}\right\rangle\right|^{2}\left|U_{n}^{l}(\rho=0)\right|^{2} S_{c v}(\hbar \omega) \\
& W_{T P} \sim\left|A_{1} A_{2}\right|^{2} \sum_{c, v}|\langle c|\varepsilon \cdot p| v\rangle|^{2} \sum_{c v}\left|\left\langle\varphi_{c} \mid \varphi_{h}\right\rangle\right|^{2}\left|\nabla U_{n}^{l}(\rho=0)\right|^{2} S_{c v}\left(\hbar \omega_{1}+\hbar \omega_{2}\right)
\end{aligned}
$$



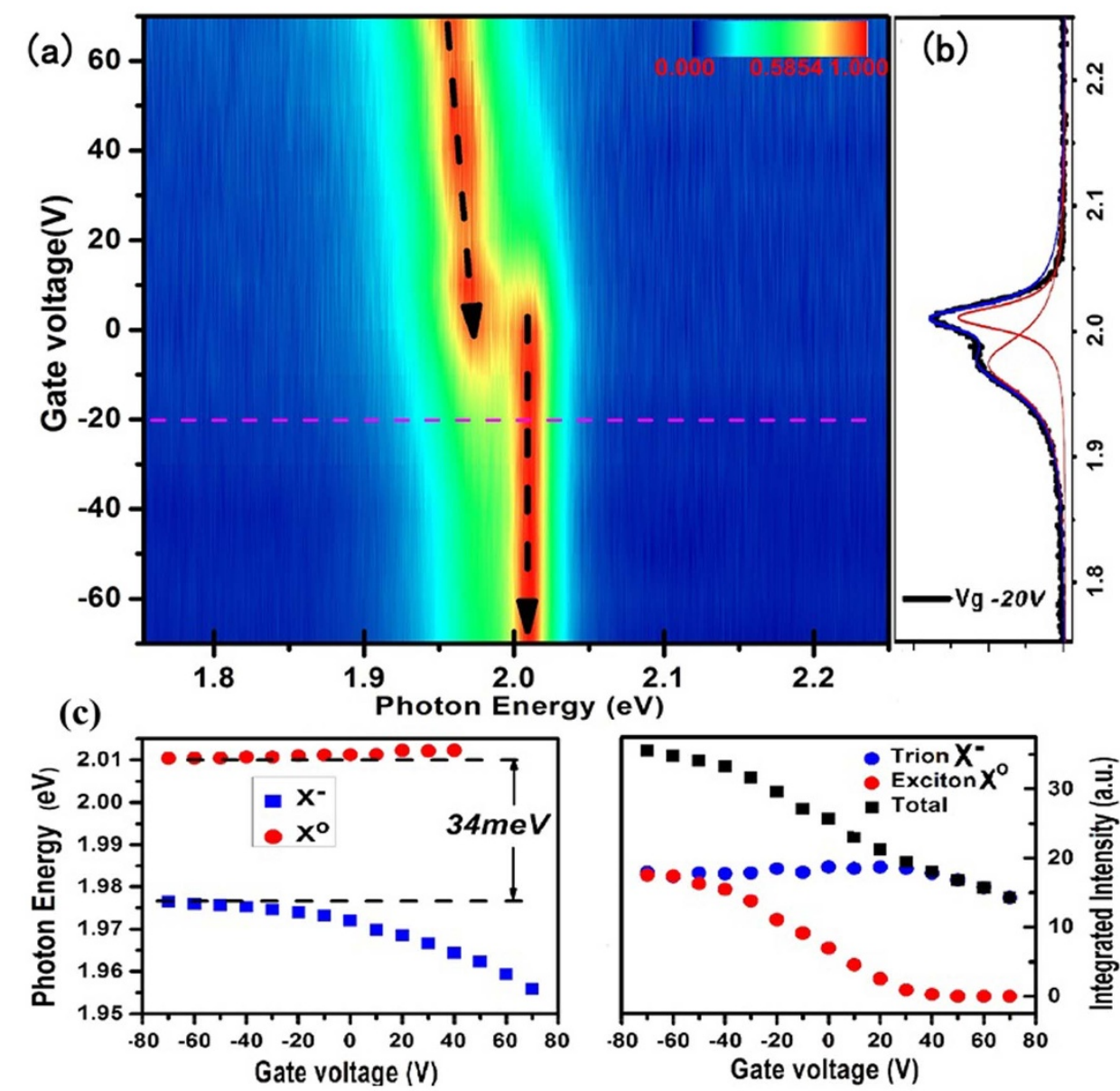

Figure 2 Electric-doping dependent photoluminescence spectra at room temperature in vacuum. (a) Colour contour plot of normalized PL spectra excited by a cw laser $(2.331 \mathrm{eV})$ under various back-gate bias. Dashed black arrows contour PL peaks of free exciton and trion states. Even at zero gate bias the trion $X^{-}$exists due to defects or substrate interactions. (b) Illustration of the PL profile at $\mathrm{V}_{\mathrm{g}}=-20 \mathrm{~V}$ (dashed line labelled in the top panel) as a superposition of two Lorentzian shape lines in red. (c) Electric gate dependence of excitons' and trions' energies (upper panel) and the corresponding integrated intensities (bottom panel).

where $A$ denotes the vector potential of the excitation, $\varepsilon$ the light polarization unit vector, $\langle c|\varepsilon \cdot p| v\rangle$ the interband matrix elements, and $S_{c v}(\hbar \omega)$ the line-shape function of interband exciton. In a $2 \mathrm{D}$ system, $U_{n}^{l}$ could be described by a solution of 2D Wannier-Mott exciton $U_{n}^{l}(\rho, \theta)=\frac{1}{\sqrt{\pi}\left(n-\frac{1}{2}\right)^{3 / 2}} \sqrt{\frac{(n-l) !}{(n+l) !}}\left(\frac{2 \rho}{n-\frac{1}{2}}\right)^{l} \exp \left(-\frac{\rho}{n-\frac{1}{2}}\right) \mathrm{L}_{\mathrm{n}-l}^{2 l}\left(\frac{2 \rho}{n-\frac{1}{2}}\right) \exp (i l \theta)$ and the exciton binding energy could be described as $E_{n}=\frac{R y^{*}}{\left(n-\frac{1}{2}\right)^{2}}$, where $\mathrm{n}=1,2 \ldots$ is the principle quantum number, $l=0,1, . .(n-1)$ is the angular quantum number, and $L_{n-l}^{2 l}$ is the associate Laguerre polynomial. As the exciton oscillator strength decays as $n^{-3}$, only the ground state $(n=1)$ and the first two excited state $(n=2)$ are considered. In a one-photon process, the ground state $1 \mathrm{~s}(n=1$, $l=0)$ dominates, whereas in a two-photon process the ground state and $n s$ states $(l=0)$ are dramatically subsidized owing to $\left|\nabla U_{n}^{l}(\rho=0)\right| \approx 0$ and the $p$ state $(l=1)$ dominates. Hence, the TPPLE usually reveals the $p$-type excited states of excitons in $2 \mathrm{D}$ systems. The one- and two-photon spectroscopy would lead to complementary information on the excitons in monolayer TMDCs.

Figure 3 shows a TP-PLE spectrum of monolayer $\mathrm{WS}_{2}$ at ambient condition, where the PL intensity of free band-edge exciton $\mathrm{A}$ is recorded as a function of the pulsed excitation energy ranging from $1.18 \mathrm{eV}$ to $1.60 \mathrm{eV}$ (limited by the output of the Ti:sapphire oscil- lator). With the contrasting optical transition strength, two-photon excitation resonant with the $p$-type excited exciton states dominates while the $s$-type states are nearly invisible. The prominent PL occurs at the excitations around $1.225 \mathrm{eV}$ and around $1.29 \mathrm{eV}$ which are absent in the linear absorption spectrum and considered as p-type states. There is a significant gap state in the range of $1.35-1.365 \mathrm{eV}$, where the PL intensity drops to be nearly negligible. The negligible but nonzero PL intensity is likely to result from the re-absorption of the second harmonic excitation, since the SHG intensity is higher than that of two-photon luminescence as shown in Figure 3(b). Upon the excitation just above the gap $(>1.365 \mathrm{eV})$ as indicated by the arrow in Figure 3(a), the PL intensity shows a linear increase with the excitation energy $(2 \hbar \omega-E g)$ as indicated in the inset. It is the signature of two-photon absorption above the band edge with in-plane polarization in $2 \mathrm{D}$ systems ${ }^{30,31}$. Besides, the two local minimums at higher excitation energy around $1.44 \mathrm{eV}(2 \hbar \omega=2.88 \mathrm{eV})$ and $1.46 \mathrm{eV}(2 \hbar \omega=2.92 \mathrm{eV})$ have significant PL intensity; therefore, they are unlikely to be the single-particle band gap state. Thus, the single particle gap or the onset of the interband continuum could be determined at $2.73 \mathrm{eV}(2 \hbar \omega=1.365 \mathrm{eV})$. Given the PL peak at $2.02 \mathrm{eV}$ presenting the energy of the ground-state exciton, the exciton binding energy of $E_{b}=0.71 \pm 0.01 \mathrm{eV}$ is extracted from the energy difference between the ground-state exciton and the onset of the inter-band continuum, which is perfectly consistent with Ref. 18 .

With the band-edge exciton binding energy of $0.71 \mathrm{eV}$, we could attribute the peaks around $1.225 \mathrm{eV}(2 \hbar \omega=2.45 \mathrm{eV})$ and $1.29 \mathrm{eV}$ 


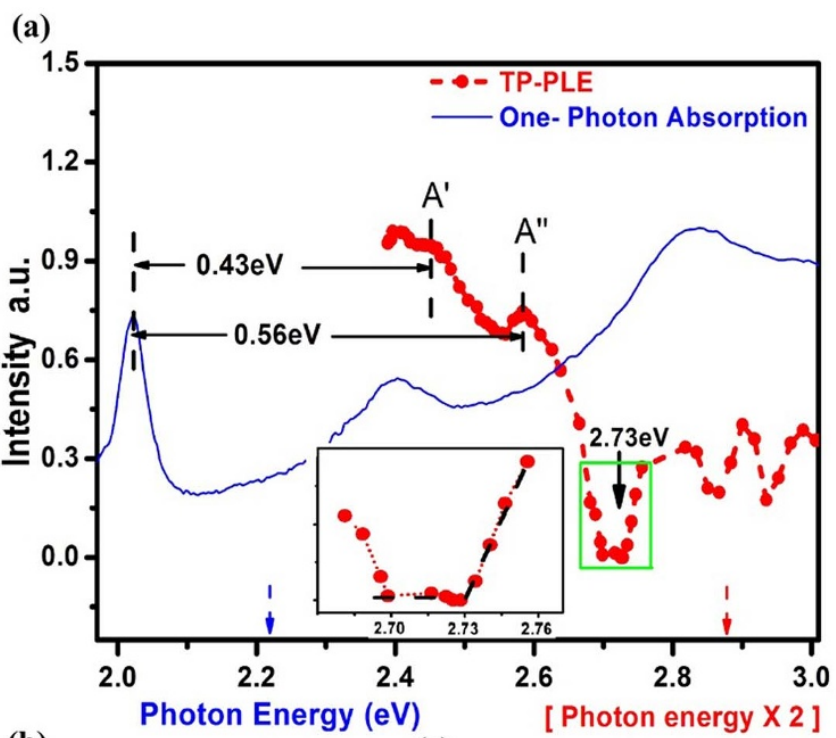

(b)

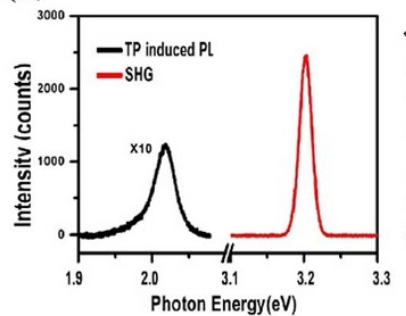

(c)

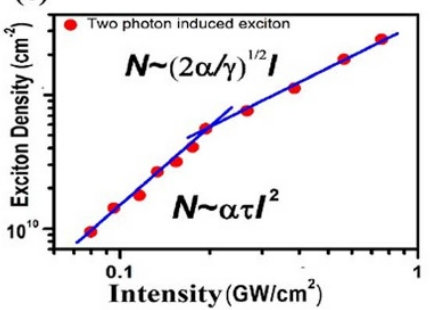

Figure 3 Two-photon photoluminescence excitation spectra at ambient condition. (a) Comparison of one-photon absorption spectrum (blue) in visible range and two-photon PLE spectrum (red) with the excitation in the range of $1.192 \sim 1.5 \mathrm{eV}$ where the $\mathrm{x}$ axis presents the exciton energy (in blue) for linear absorption and the double of the excitation energy (in red) for TP-PLE. Both spectra are obtained at ambient conditions. $\mathrm{A}^{\prime}$ and $\mathrm{A}^{\prime \prime}$ denote the excited states of exciton A and the zoom-in of the gap state section is shown in inset. The TP-PL intensity linearly increases with the excitation energy just above the threshold of the interband continuum, presenting the signature of two-photon process in $2 \mathrm{D}$ systems where the polarization sits in the $2 \mathrm{D}$ plane. (b) The spectra of TP-PL and the second harmonic generation (SHG) at the excitation of $1.6 \mathrm{eV}$. The integrated intensity of the PL is more than one order of magnitude less than that of the SHG. (c) The intensity of ground state exciton vs. the excitation intensity under the excitation of $1.59 \mathrm{eV}$. The fitting lines demonstrate a quadratic(under low intensity) and a linear-dependence (under high intensity) respectively, which yield the exciton-exciton annihilation rate $\gamma \approx 0.31-$ $0.47 \mathrm{~cm}^{2} / \mathrm{s}$ and the two-photon absorption cross section $\alpha \approx 3.5-5.3 \times$ $10^{4} \mathrm{~cm}^{2} \mathrm{~W}^{-2} \mathrm{~S}^{-1}$.

$(2 \hbar \omega=2.58 \mathrm{eV})$ in the TP-PLE spectrum to the excited states of excitons, which are qualitatively consistent with the recent $a b$ initio calculation and the experimental findings ${ }^{18}$. As the exciton A and $\mathrm{B}$ both originate from the spin-split valence bands at $K\left(K^{\prime}\right)$ valley with the similar effective mass, a similar strength of binding energy is expected. Besides, the PL intensity around the peak $\mathrm{A}^{\prime}$ and $\mathrm{A}^{\prime \prime}$ monotonically decreases. Both peaks are likely to be the p-type excited states of the same exciton. Due to the limit of the light source in the experiment, we could not probe the states in the range of 2.0$2.4 \mathrm{eV}$. If no p-type exciton state appears in the energy of $2.0-2.4 \mathrm{eV}$, the peak $A^{\prime}$ and $A^{\prime \prime}$ could be tentatively assigned to the $2 p$ and $3 p$ states of exciton $A$, respectively. Otherwise, the peak $A^{\prime}$ and $A^{\prime \prime}$ will be assigned to $3 p$ and $4 p$ states. The exciton binding energy could also be evaluated from the energy difference between exciton $1 s$ and $n p$ states. The $2 \mathrm{D}$ hydrogen model gives the energy difference between $1 s$ and $2 p(3 p)$

$$
E_{b}=4 R_{y}^{*}=\frac{9}{8} \Delta E_{1 s-2 p}=\frac{100}{96} \Delta E_{1 s-3 p}=\frac{49}{48} \Delta E_{1 s-4 p}
$$

which corresponds to $E_{b}=0.48 \mathrm{eV}$ and $E_{b}=0.58 \mathrm{eV}$, respectively. The alternative assignment, for example, peak $\mathrm{A}^{\prime}$ and $\mathrm{A}^{\prime \prime}$ to $3 p$ and $4 p$ states, leads to $E_{b}=0.45 \mathrm{eV}$ and $0.57 \mathrm{eV}$ (According to Ref. 18, the $\mathrm{A}^{\prime}$ and $\mathrm{A}^{\prime \prime}$ are assigned to $3 \mathrm{p}$ and $4 \mathrm{p}$ excited states of $\mathrm{A}$ excitons, respectively).

These are significantly smaller than $E_{b}=0.71 \pm 0.01 \mathrm{eV}$ extracted from the energy difference between the ground state exciton and the onset of the interband continuum The distribution of these excited states also significantly deviates from that of the $2 \mathrm{D}$ hydrogen model. The difference may lie in the modification of the $2 \mathrm{D}$ hydrogen model by electron-phonon and electron correlation interactions in monolayer TMDCs. The recent first principle simulation shows that qdependent screening dramatically enhances the binding energy of the excited states of excitons ${ }^{5,18}$. Nevertheless, it is safe to extract the exciton binding energy of $E_{b}=0.71 \pm 0.01 \mathrm{eV}$ by the energy difference between $1 s$ exciton and the onset of the interband continuum, independent of the assignments of the excited states. There exist discrepancies in measurements of exciton binding energy ${ }^{18-24}$. Besides, controversial results on exciton binding energy of $\mathrm{MoSe}_{2}$ monolayers have been reported by scanning tunneling spectroscopy ${ }^{23}$ and angle-resolved photoemission spectroscopy ${ }^{32}$. One possible cause may lie in the difference of samples. A recent theoretical calculation shows that the high doping could significantly modify the exciton binding energy and the optical band gap in monolayer $\mathrm{TMDCs}^{33}$.

The two-photon absorption has a quadratic dependence on the excitation intensity in principle. The two-photon induced photoluminescence (TP-PL) intensity from monolayer $\mathrm{WS}_{2}$ displays a clear quadratic dependence on the excitation intensity at low excitation intensity as shown in Figure 3(c). As the excitation intensity increases above $0.2 \mathrm{GW} / \mathrm{cm}^{2}$, the PL intensity experiences a clear transition from a quadratic dependence to a linear dependence on the excitation intensity. The linear dependence may result from some nonradiative channels arising from the exciton-exciton interactions when the exciton density increases to a certain level. If we follow the simple model

$$
\frac{d N}{d t}=\alpha I^{2}-\frac{N}{\tau}-\frac{1}{2} \gamma N^{2}=0
$$

where $N$ denotes the exciton density, $I$ the excitation intensity, $\alpha$ the two-photon absorption cross section, $\tau$ the exciton lifetime and $\gamma$ the exciton-exciton annihilation rate, the fitting of the quadratic dependence $I_{p h}=\alpha \tau I^{2}(I \rightarrow 0)$ gives two-photon absorption cross section of $\alpha$ $\approx 3.5-5.3 \times 10^{4} \mathrm{~cm}^{2} \mathrm{~W}^{-2} \mathrm{~S}^{-1}$ at $1.59 \mathrm{eV}$ where the PL quantum yield of $4 \times 10^{-3}$ and the exciton lifetime of 100 ps are assumed ${ }^{34}$. Subsequently, the linear dependence slope at high intensity $\sqrt{\frac{2 \alpha}{\gamma}}$ yields the exciton-exciton annihilation rate $\gamma \approx 0.31-0.47 \mathrm{~cm}^{2} / \mathrm{s}$, which is qualitatively consistent with that in monolayer $\mathrm{MoSe}_{2}$ measured by pump-probe reflection spectroscopy ${ }^{35}$. The linear intensity dependence of TP-PL is the evidence of the strong exciton-exciton interactions in monolayer TMDC.

In summary, the linear absorption spectroscopy cannot resolve the electronic interband transition edge down to $10 \mathrm{~K}$, due to the strong electron-phonon scattering and the overlap of excitons around $\Gamma$ point and the transfer of the oscillator strength from interband continuum to exciton states. The TP-PLE measurements successfully probe the excited states of the band-edge exciton and the single-particle band gap. The exciton binding energy of $0.71 \pm 0.01 \mathrm{eV}$ is extracted by the energy difference between 1s exciton and the single-particle gap in monolayer $\mathrm{WS}_{2}$. The distribution of the exciton excited states significantly deviates from the $2 \mathrm{D}$ hydrogen model. The giant exciton binding 
energy manifests the unprecedented strong Coulomb interactions in monolayer TMDCs.

1. Brus, L. E. Electron-electron and electron-hole interactions in small semiconductor crystallites: The size dependence of the lowest excited electronic state. J. Chem. Phys 80, 4403-4409 (1984).

2. Wang, F., Dukovic, G., Brus, L. E. \& Heinz, T. F. The optical resonances in carbon nanotubes arise from excitons. Science 308, 838-841 (2005).

3. Maultzsch, J. et al. Exciton binding energies in carbon nanotubes from twophoton photoluminescence. Phys. Rev. B 72, 241402(R) (2005).

4. Cheiwchanchamnangij, T. \& Lambrecht, W. R. L. Quasiparticle band structure calculation of monolayer, bilayer, and bulk MoS2. Phys. Rev. B 85, 205302 (2012).

5. Qiu, D. Y., da Jornada, F. H. \& Louie, S. G. Optical Spectrum of $\mathrm{MoS}_{2}$ : Many-Body Effects and Diversity of Exciton States. Phys. Rev. Lett. 111, 216805 (2013).

6. Komsa, H.-P. \& Krasheninnikov, A. Effects of confinement and environment on the electronic structure and exciton binding energy of $\mathrm{MoS}_{2}$ from first principles. Phys. Rev. B 86, 241201(R) (2012).

7. Shi, H. L., Pan, H., Zhang, Y. W. \& Yakobson, B. I. Quasiparticle band structures and optical properties of strained monolayer $\mathrm{MoS}_{2}$ and $\mathrm{WS}_{2}$. Phys. Rev. B 87, 155304 (2013).

8. Ramasubramaniam, A. Large excitonic effects in monolayers of molybdenum and tungsten dichalcogenides. Phys. Rev. B 86, 115409 (2012).

9. Mak, K. F., Lee, C., Hone, J., Shan, J. \& Heinz, T. F. Atomically Thin $\mathrm{MoS}_{2}$ : A New Direct-Gap Semiconductor. Phys. Rev. Lett. 105, 136805 (2010).

10. Splendiani, A. et al. Emerging Photoluminescence in Monolayer $\mathrm{MoS}_{2}$. Nano Lett. 10, 1271-1275 (2010).

11. Zeng, H. et al. Optical signature of symmetry variations and spin-valley coupling in atomically thin tungsten dichalcogenides. Sci. Rep. 3, 1608; DOI:10.1038/ srep01608 (2013).

12. Zhao, W. et al. Evolution of Electronic Structure in Atomically Thin Sheets of WS and WSe $\mathrm{W}_{2}$. ACS Nano 7, 791-797 (2012).

13. Bordas, J. [Some Aspects of Modulation Spectroscop in Optical and electrical properties] Optical and Electrical Properties, [Lee, P. (ed)][145-229] (Springer Netherlands, 1976)

14. Mak, K. F. et al. Tightly bound trions in monolayer $\mathrm{MoS}_{2}$. Nat. Mater. 12, 207-211 (2013).

15. Ross, J. S. et al. Electrical control of neutral and charged excitons in a monolayer semiconductor. Nat. Commun. 4, 1474 (2013).

16. Mitioglu, A. et al. Optical manipulation of the exciton charge state in single-layer tungsten disulfide. Phys. Rev. B 88, 245403 (2013).

17. Thilagam, A. Two-dimensional charged-exciton complexes. Phys. Rev. B 55, 7804-7808 (1997)

18. Ye, Z. et al. Probing excitonic dark states in single-layer tungsten disulphide. Nature 513, 214-218 (2014).

19. He, K. et al. Tightly Bound Excitons in Monolayer WSe ${ }_{2}$. Phys. Rev. Lett. 113, 026803 (2014).

20. Chernikov, A. et al. Exciton Binding Energy and Nonhydrogenic Rydberg Series in Monolayer $\mathrm{WS}_{2}$. Phys. Rev. Lett. 113, 076802 (2014).

21. Wang, G. et al. Non-linear Optical Spectroscopy of Excited Exciton States for Efficient Valley Coherence Generation in WSe ${ }_{2}$ Monolayers. arXiv: 1404.0056 (2014).

22. Zhang, C., Johnson, A., Hsu, C.-L., Li, L.-J. \& Shih, C.-K. Direct Imaging of the Band Profile in Single Layer MoS2 on Graphite: Quasiparticle Energy Gap, Metallic Edge States and Edge Band Bending. Nano Lett. 14, 2443-2447 (2014).

23. Ugeda, M. M. et al. Giant bandgap renormalization and excitonic effects in a monolayer transition metal dichalcogenide semiconductor. Nat. Mater. 13 1091-1095 (2014).
24. Hanbicki, A. T., Currie, M., Kioseoglou, G., Friedman, A. L. \& Jonker, B. T. Measurement of high exciton binding energy in the monolayer transition-metal dichalcogenides WS2 and WSe2. Solid State Commun. 203, 16-20 (2015).

25. Reina, A. et al. Transferring and Identification of Single- and Few-Layer Graphene on Arbitrary Substrates. J. Phys. Chem. C 112, 17741-17744 (2008).

26. Perebeinos, V., Tersoff, J. \& Avouris, P. Electron-Phonon Interaction and Transport in Semiconducting Carbon Nanotubes. Phys. Rev. Lett. 94, 086802 (2005).

27. Gong, Z. et al. Magnetoelectric effects and valley-controlled spin quantum gates in transition metal dichalcogenide bilayers. Nat. Commun. 4, 2053 (2013).

28. Tongay, S. et al. Broad-Range Modulation of Light Emission in Two-Dimensional Semiconductors by Molecular Physisorption Gating. Nano Lett. 13, 2831-2836 (2013).

29. Xiao, D., Liu, G.-B., Feng, W., Xu, X. \& Yao, W. Coupled Spin and Valley Physics in Monolayers of MoS2 and Other Group-VI Dichalcogenides. Phys. Rev. Lett. 108, 196802 (2012).

30. Shimizu, A., Ogawa, T. \& Sakaki, H. Two-photon absorption spectra of quasi-lowdimensional exciton systems. Phys. Rev. B 45, 11338 (1992).

31. Tai, K., Mysyrowicz, A., Fischer, R. J., Slusher, R. E. \& Cho, A. Y. Two-photon absorption spectroscopy in GaAs quantum wells. Phys. Rev. Lett. 62, 1784 (1989).

32. Zhang, Y. et al. Direct observation of the transition from indirect to direct bandgap in atomically thin epitaxial MoSe2. Nat. Nanotechnol. 9, 111-115 (2013).

33. Zhang, C., Wang, H., Chan, W., Manolatou, C. \& Rana, F. Absorption of light by excitons and trions in monolayers of metal dichalcogenide MoS2:Experiments and theory. Phys. Rev. B 89, 205436 (2014).

34. Zhu, B., Zeng, H., Dai, J., Gong, Z. \& Cui, X. Anomalously robust valley polarization and valley coherence in bilayer WS2. Proc. Natl. Acad. Sci. U.S.A. 111, 11606-11611 (2014).

35. Kumar, N., Cui, Q., Ceballos, F., He, D., Wang, Y. \& Zhao, H. Exciton-exciton annihilation in MoSe2 monolayers. Phys. Rev. B 89, 125427 (2014).

\section{Acknowledgments}

The work is supported by Area of excellency (AoE/P-04/08), CRF of Hong Kong Research Grant Council(HKU9/CRF/13G) and SRT on New Materials of The University of Hong Kong.

\section{Author contributions}

X.D.C. conceived the experiments; B.R.Z. and X.C. carried out the experiments and data analysis; All authors discussed and wrote the manuscript.

\section{Additional information}

Supplementary information accompanies this paper at http://www.nature.com/ scientificreports

Competing financial interests: The authors declare no competing financial interests.

How to cite this article: Zhu, B., Chen, X. \& Cui, X. Exciton Binding Energy of Monolayer $\mathrm{WS}_{2}$. Sci. Rep. 5, 9218; DOI:10.1038/srep09218 (2015).

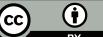

This work is licensed under a Creative Commons Attribution 4.0 International License. The images or other third party material in this article are included in the article's Creative Commons license, unless indicated otherwise in the credit line; if the material is not included under the Creative Commons license, users will need to obtain permission from the license holder in order to reproduce the material. To view a copy of this license, visit http://creativecommons.org/licenses/by/4.0/ 